\title{
Resistance training interventions across the cancer control continuum: a systematic review of the implementation of resistance training principles
}

\author{
C M Fairman, P N Hyde, B C Focht
}

\begin{abstract}
- Additional material is published online only. To view please visit the journal online (http://dx.doi.org/10.1136/ bjsports-2016-096537).
\end{abstract}

Exercise and Behavioral Medicine Laboratory, Kinesiology, The Ohio State University, Columbus, Ohio, USA

\section{Correspondence to}

Ciaran Fairman, Exercise and Behavioral Medicine Laboratory, Kinesiology, The Ohio State University, 305 W 17th Avenue, Columbus, OH 43201, USA; Fairman.13@osu.edu

Accepted 30 November 2016 Published Online First 16 December 2016
CrossMark

To cite: Fairman $C M$, Hyde PN, Focht BC. Br J Sports Med 2017:51:677-685.
ABSTRACT

Objectives The primary purpose of this systematic review is to examine the extant resistance training (RT) cancer research to evaluate the proportion of RT interventions that: (1) implemented key RT training principles (specificity, progression, overload) and (2) explicitly reported relevant RT prescription components (frequency, intensity, sets, reps).

Design A qualitative systematic review was performed by two reviewers (CMF and PNH) who inspected the titles and abstracts to determine eligibility for this systematic review. Identified papers were obtained in full and further reviewed. Data were extracted to evaluate the application of principles of training, along with specific RT components.

Data sources Electronic databases (PubMed, EMBASE, CINAHL, Cochrane, PEDro, Psychlnfo, Cancer Lit, Sport Discus, AMED, Cochrane Central Register of Controlled Trials) and reference lists of included articles from inception to May 2016.

Results 37 studies were included. The principle of specificity was used appropriately in all of the studies, progression in $65 \%$ and overload in $76 \%$ of the studies. The most common exercise prescription ( $50 \%)$ implemented in the studies included in this review were 2-3 days/week, focusing on large muscle groups,

$60-70 \% 1$ repetition maximum (RM), 1-3 sets of $8-12$ repetitions.

Conclusions Reporting of RT principles in an oncology setting varies greatly, with often vague or non-existent references to the principles of training and how the RT prescription was designed.

\section{INTRODUCTION}

To date, the prevailing evidence in the field of exercise oncology supports the safety and efficacy of exercise as a means to attenuate many of the treatment-related adverse effects, such as risk for cardiovascular disease, increased fatigue and diminished physical functioning and quality of life. ${ }^{1-11}$ Moreover, the culmination of the extant literature supporting the benefits of exercise for patients with cancer and cancer survivors has resulted in the release of several position stands and expert statements from numerous international agencies advocating physical activity in this population. ${ }^{12-15}$ These recommendations provide beneficial guidance in developing safe and effective exercise programming for patients with cancer and cancer survivors. However, the exercise response in a patient population with cancer can vary based on the cancer type, treatment type, dose and duration, and time along the cancer continuum. Consequently, it is plausible that applying a generic resistance training (RT) prescription to a heterogeneous patient population with cancer may unintentionally mask the full therapeutic potential of RT as a supportive care intervention in the treatment of cancer. $^{16}$

The development and implementation of RT programmes are traditionally guided by key RT principles such as specificity (training stimulus must be specific to the desired outcome), progression (training stimulus must be systematically progressed to provide a greater than normal stress) and overload (greater than normal stress must occur for adaptation to occur). ${ }^{17}{ }^{18}$ Implementation of these training principles are used to systematically guide the manipulation of primary components of the RT stimulus including duration, load and volume. Consequently, given that the application of these training principles is integral to optimising the improvements accompanying RT observed across the cancer control continuum, determining the extent to which key scientific principles of RT (ie, specificity, progression and overload) (table 1) are implemented in RT interventions is an integral consideration for guiding future exercise prescription approaches designed for patients with cancer and cancer survivors.

There is a critical need for the scientific principles of RT and the components of the RT stimulus, to be applied rigorously and reported in detail in RT prescription to allow for appropriate evaluation of the feasibility, efficacy and effectiveness of RT interventions in a patient population with cancer. Careful attention to the implementation of these principles and reporting of RT stimulus components is important to accurately evaluate the therapeutic efficacy of RT as a supportive care intervention across the cancer control continuum. ${ }^{19} 20$ Accordingly, a thorough evaluation of the individual components of the exercise prescription including frequency, intensity, time, sets and reps is also warranted.

Previous reviews have examined interventions with regard to their application of common principles of exercise training across various patient populations with cancer. ${ }^{19}{ }^{20}$ However, to the best of our knowledge, a comprehensive review focusing specifically on the application of RT principles and RT stimulus components/prescription has yet to be conducted. We contend that determining the extent to which RT principles have been implemented in interventions is an important objective given the present state of knowledge of RT prescription 
Table 1 Exercise training principles and description of RT components

\begin{tabular}{|c|c|}
\hline Principle and definition & Criteria for this review \\
\hline $\begin{array}{l}\text { Specificity: Training should be specific } \\
\text { to muscles trained, and be relevant to } \\
\text { desired outcomes }\end{array}$ & $\begin{array}{l}\text { The intervention is designed to induce } \\
\text { improvements in a primary outcome }\end{array}$ \\
\hline $\begin{array}{l}\text { Progression: The amount of load or } \\
\text { resistance must be increased, } \\
\text { providing a greater stress than the } \\
\text { body is used to for continuous } \\
\text { adaptation }\end{array}$ & $\begin{array}{l}\text { The intervention gradually increased in } \\
\text { frequency, sets, reps and/or load over } \\
\text { the course of the intervention }\end{array}$ \\
\hline $\begin{array}{l}\text { Overload: Greater than normal stress } \\
\text { must occur for fitness to improve }\end{array}$ & $\begin{array}{l}\text { Interventions included baseline testing } \\
\text { to determine exercise intensity (ie, } \\
1 \mathrm{RM} \text { ), or rationale that intervention } \\
\text { was of sufficient intensity }\end{array}$ \\
\hline Component of RT prescription & Description \\
\hline Frequency & How many days per week \\
\hline Intensity & Either per cent $1 \mathrm{RM}, \mathrm{RPE}$ or RM \\
\hline Time & Duration of session \\
\hline Sets & $\begin{array}{l}\text { How many sets of each exercise were } \\
\text { performed }\end{array}$ \\
\hline Repetition range & $\begin{array}{l}\text { What was the repetition range for each } \\
\text { set }\end{array}$ \\
\hline Exercise selection & $\begin{array}{l}\text { An outline of the exercises used for the } \\
\text { intervention }\end{array}$ \\
\hline
\end{tabular}

in cancer care. Evaluating the extent to which key RT principles have been implemented is an important, yet presently understudied, aspect that may influence the utility of implementing RT in supportive care of cancer.

\section{OBJECTIVE}

The primary purpose of this systematic review is to examine the extant RT-cancer research to evaluate the proportion of RT interventions that: (1) implemented key RT training principles (specificity, progression, overload) and (2) explicitly reported relevant RT prescription components (frequency, intensity, sets, reps, etc).

\section{METHODS}

\section{Design}

This was a qualitative systematic review. Before conducting searches of any kind, a priori eligibility criteria were established and applied to the search yield.

\section{Eligibility criteria for selecting studies}

We assessed randomised controlled trials (RCTs) for eligibility for inclusion. To be included, RCTs must have examined a RT intervention in isolation, and included adult patients with cancer or cancer survivors who were actively undergoing cancer treatment or had cancer treatment with curative intent, and have been published in English in a peer-reviewed journal.

\section{Data sources}

Scientific databases (PubMed, EMBASE, CINAHL, Cochrane, PEDro, PsychInfo, Cancer Lit, Sport Discus, AMED, Cochrane Central Register of Controlled Trials) were searched in February 2016. A subsequent search was performed in May 2016 to ensure inclusion of any additional articles that were published in the interim. Search terms related to cancer (cancer, oncology, tumour, malignancy, chemotherapy) and RT (weight training, RT, strength training) were entered in different combinations as
Medical Subject Heading terms and keywords where appropriate. All terms were applied to every database and the search strategy as applied to PubMed can be found in online supplementary appendix 1. Manual searches were also conducted using the reference lists of other narrative and meta-analytic review of the exercise oncology literature. CMF performed the initial search using the search terms in all possible combinations which was then confirmed by PNH.

\section{Article selection}

Two authors (CMF and PNH) independently screened the title and abstract of every citation found in the literature search. Two lists were compared to achieve consensus for eligibility. Identified papers of relevance were then obtained in full and the eligibility criteria were applied independently by both authors. Any discrepancies were resolved by discussion and consensus with an independent reviewer (BCF). Consistent with PRISMA guidelines, the flow diagram in figure 1 outlines the results of the computerised searches.

\section{Data extraction}

Data extraction guidelines were developed to systematically extract data from each study under the following headings: the population studied, cancer treatment (if applicable), total sample size, outcomes of interest, exercise programme details such as frequency, intensity, set, reps, etc, and incorporation of training principles such as overload and progression.

Relevant details of each study included in the study were extracted and rated independently by both authors according to each principle of training. Any discrepancies were settled via discussion among all authors (CMF, $\mathrm{PNH}$ and $\mathrm{BCF})$. Incorporation of principles was determined by a study having detail on their intervention that fell within the operational definition of each principle outlined in table 1 . Incorporation and/or reporting of the principle was assigned a 'Y', whereas 'UC' was assigned if it was unclear whether or not the principle was used, or if it was used but inconsistently applied (eg, an intervention progressed the load to a point, then discontinued progression). 'NR' was assigned if a principle was not reported in the intervention. Details if/how and the level to which principles of progression and overload were reported in each study were characterised by one of the following statements: (1) no mention of if/how principle was incorporated, (2) mentioned the principle, but no description of how it was incorporated, (3) brief, but vague description of incorporation of principle and (4) clear description of incorporation of principle. The reporting score for each principle is outlined in table 2 . Additionally, the specific details of each RT intervention have been outlined in supplementary appendix 2. Characteristics of each intervention according to the FITT principle, frequency (how often), intensity (prescribed intensity of the activity), time (duration of each bout) and type (excluded as all studies included were RT interventions), along with details on prescribed sets, repetition ranges and exercises were extracted. An overview of the exercise training principles and the description of RT components are provided in table 1 .

\section{Risk of bias}

The Physiotherapy Evidence Database (PEDro) scale was used to assess the risk of bias in included studies The PEDro scale rates internal study validity and the presence of statistical replicable information on a scale from 0 (high risk of bias) to 10 (low risk of bias) with $\geq 6$ representing a cut-off score for studies with low risk of bias. $^{21}$ 


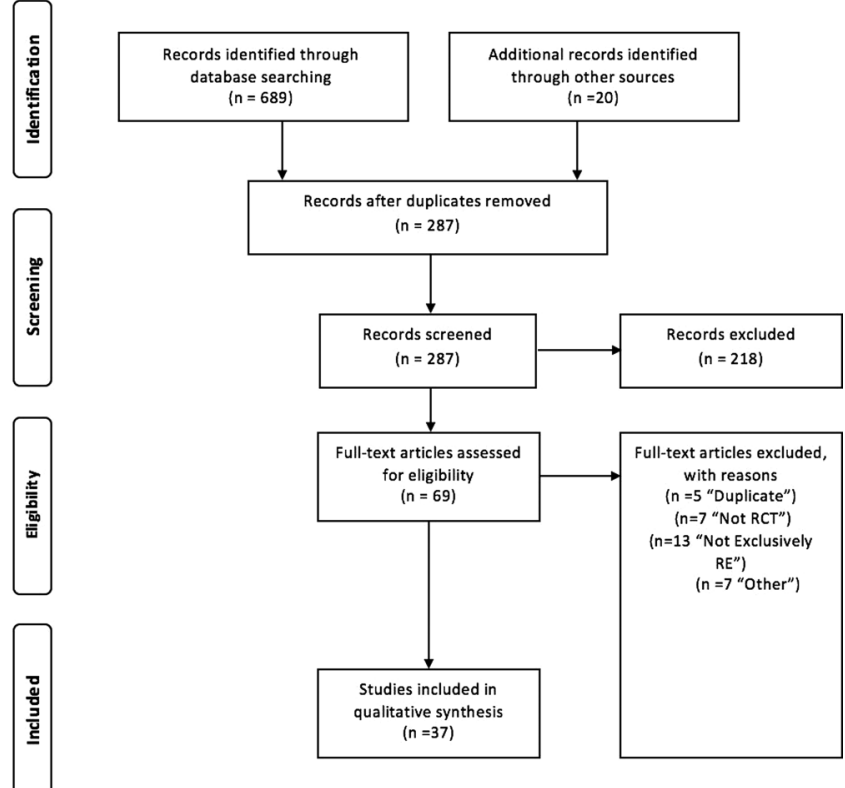

Figure 1 PRISMA flow diagram.

\section{Analyses}

A summary of findings for each study was compiled to depict sample characteristics, number of participants, study duration and outcomes of interest (table 2). A summary of the application of key training principles, the RT stimulus components of sets, reps and intensity used, as well as an outline of the exercises in the RT intervention session, is compiled into table 2 . The proportion of interventions that applied each RT principle and explicitly reported the RT stimulus components was transformed into percentages via proportion of studies using a principle relative to the total number of studies included in this review.

\section{RESULTS}

\section{Study characteristics}

An outline of the study design, participant and intervention characteristics are provided in table 3. A total of 709 records were identified by the literature search, 69 of which were selected for full-text review $(\kappa=0.68)$. PEDro scores from the included studies ranged from 5 to 9 . Items that were fulfilled by the majority of studies were 'eligibility criteria' $(\mathrm{n}=41)$, 'random allocation' $(\mathrm{n}=41)$, 'no baseline difference' $(\mathrm{n}=38)$, 'between-groups statistical outcome' $(n=37)$, and 'point measures of variability' $(n=41)$. Roughly half of the studies concealed allocation $(n=23)$, or used the intention to treat principle in their analysis $(\mathrm{n}=25)$. A majority of the studies failed with blinding of subjects $(\mathrm{n}=0)$ and assessors $(\mathrm{n}=15)$. Individual PEDro scores for each study are presented in table 3. A total of 37 studies involving 3251 participants met the inclusion criteria of the review $(\kappa=0.87)$. A total of 18 studies were conducted during adjuvant treatment (chemotherapy and/or radiation), whereas 19 studies were conducted after the completion of treatment. The length of the interventions ranged from 9 weeks to 52 weeks. Few studies in the review targeted one outcome measure as their primary outcome, with many aiming to improve a variety of physiological and psychosocial outcomes. Thus, all of the studies' outcomes are included in outcomes of interest in table 3. Figure 1 outlines the results of the searches and full-text reviews. Following full-text review, 32 studies were excluded for the following reasons: duplicate $(n=9),{ }^{22-30}$ not RCT $(\mathrm{n}=6),{ }^{31-36}$ not exclusively RT $(\mathrm{n}=10),{ }^{63-45}$ other $(\mathrm{n}=7) .{ }^{46-52}$

\section{Application of RT principles}

An overview of the application of RT principles is provided in table 4 . The principle of specificity was used appropriately by all of the studies (100\%) by matching the intervention to the study objective (body composition, strength, physical function, lymphoedema symptoms, etc). The principle of progression was applied appropriately in 24 of the $37(65 \%)$ studies. The principle was unclear or incorrectly applied in the remaining 13 (35\%) studies. The principle of overload was applied appropriately by $28(76 \%)$ of the 37 studies. The application of this principle was unclear or non-existent in the remaining nine (24\%) studies.

\section{Reporting of RT components}

All studies (100\%) included in this review reported the frequency of sessions. Frequency ranged from 1 to 4 sessions per week. All but one (98\%) of the studies reported the intensity of the exercise prescription. The intensity was prescribed either using a percentage of 1 repetition maximum (RM), or a target RM for a given session (eg, 8-10 RM). The duration of the entire RT intervention was reported in all studies (100\%). Additionally, the duration of each RT session was reported by $13(40 \%)$ studies. All studies (100\%) reported the number of sets performed during the intervention. Sets performed during a given session ranged from 1 to 4 . All studies reported the repetitions range prescribed during the intervention. The reps prescribed ranged from 6 to 20 . The most commonly reported prescription $(\sim 50 \%)$ included in the review was $2-3$ days/week, $1-3$ sets, $8-12$ reps at $60-80 \%$ of $1 \mathrm{RM}$, focusing on machinebased exercises for muscles of the chest, back, shoulders, arms, buttock, hips, thighs and calves.

\section{DISCUSSION}

The primary purposes of this systematic review were to examine the extant RT-cancer literature to evaluate the proportion of RT interventions that: (1) implemented key RT principles (specificity, progression, overload) and (2) explicitly reported relevant RT prescription components (frequency, intensity, sets, reps, etc). A total of 37 studies examining RT in patient populations with cancer were included in this review. The principles of specificity, progression and overload were incorporated in $100 \%$, $65 \%$ and $76 \%$ of the studies, respectively. Thus, there is meaningful variability in the implementation of the key RT principles of progression and overload across RT trials among patients with cancer and cancer survivors. Additionally, the majority of studies explicitly reported the relevant RT prescription components. The most common exercise prescription ( $50 \%)$ implemented in the studies included in this review were 2-3 days/ week, focusing on large muscle groups, $60-70 \% 1 \mathrm{RM}, 1-3$ sets of 8-12 repetitions. Taken collectively, the findings of the present review have important implications for RT prescription approaches as well as the design and delivery of future RT interventions targeting patient populations with cancer.

\section{Incorporation of RT principles}

The principle of specificity was incorporated by all of the studies included in the review. All of the trials appropriately matched the RT intervention to a desired outcome of muscular strength, physical function or lymphoedema status. This is consistent with prior reviews of the exercise oncology literature reporting that the principle of specificity was reported in all RT studies. ${ }^{19} 20$ The incorporation of this principle ensures the most appropriate mode of exercise is selected to optimise the 


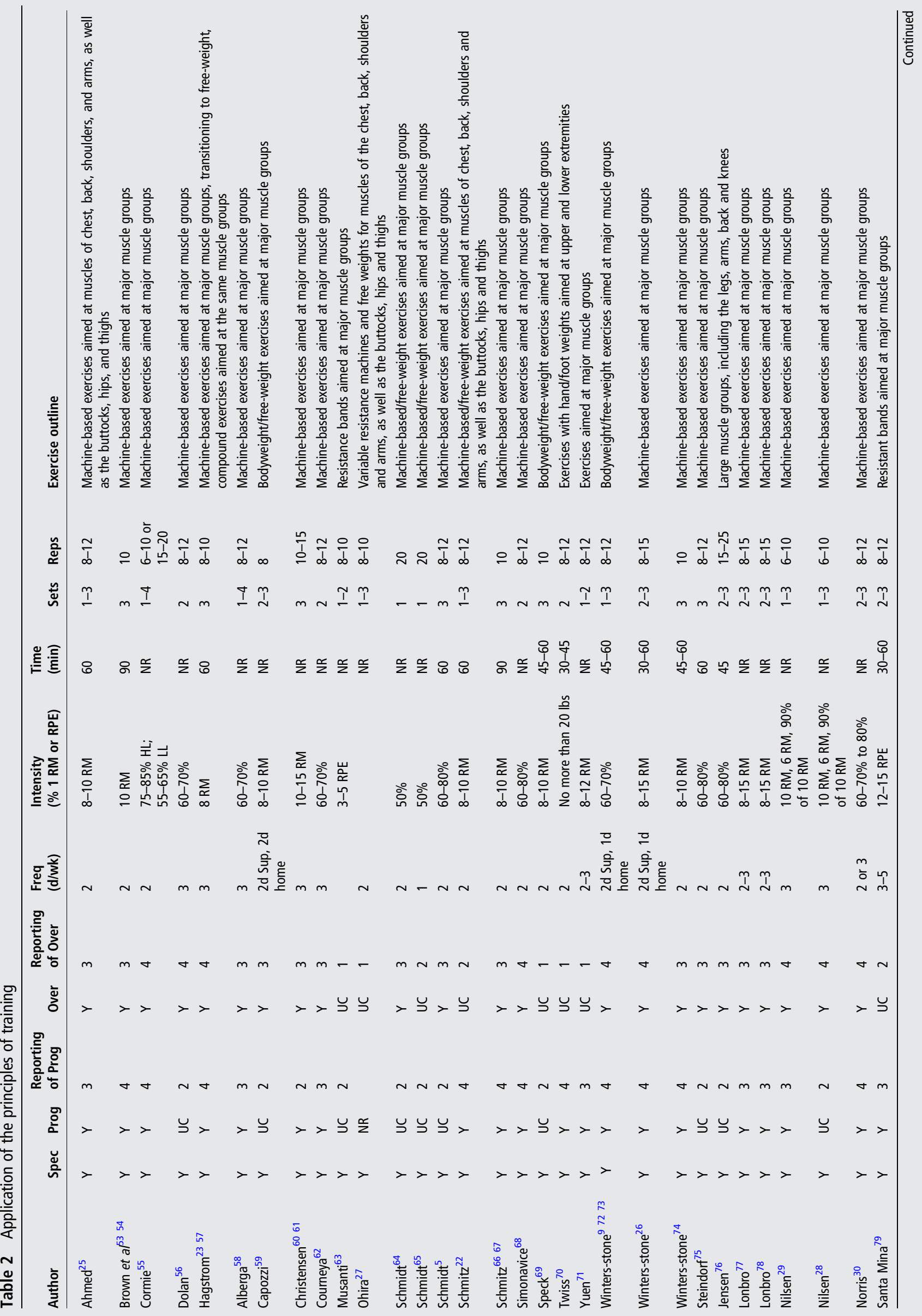




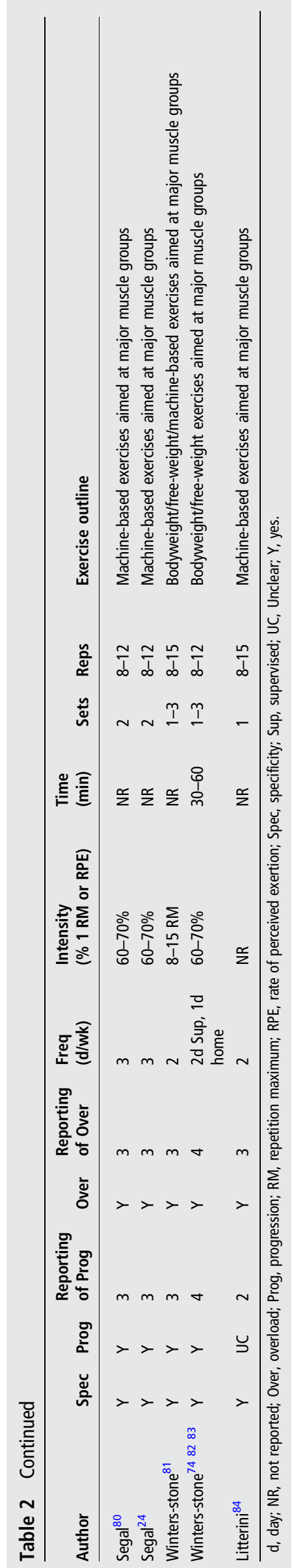

desired outcome. Furthermore, incorporating the specificity principle facilitates the likelihood that improvements in the outcomes of interest are attributable to the effects of the RT intervention.

The principle of overload was not incorporated in $24 \%$ of the interventions included in this review. The principle of overload states that a muscle must act against greater than normal resistance to ensure appropriate stimulation and adaptation. Most commonly in RT, overload can be expressed a percentage of $1 \mathrm{RM}$, or a RM range (ie, $8-12$ reps). Failure to ensure that exercise provides an adequate stimulus to the muscle can lead to minimal, if any, improvements in the desired outcome. Overload was not incorporated by studies either failing to conduct a baseline assessment of strength to determine an initial training stressor, or providing an initial load that could be deemed insufficient relative to current standards. For example, a study included in this review began participants with no weight or 1-pound weights for their initial weeks. ${ }^{74}$ While this can be appreciated as a safe approach to loading, it can also be interpreted as an overly conservative, potentially ineffective loading protocol that may undermine the extent to which the RT intervention will produce meaningful improvements in clinically relevant outcomes. A higher initial loading may have led to even greater improvements in the desired outcomes. Thus, while the load prescribed in many of the interventions may have been effective, the results should be interpreted with the consideration that optimal loading stimulus may not have been implemented in at least $24 \%$ of the trials.

The principle of progression was insufficiently reported in $35 \%$ of the interventions included in the review. These studies provided either very little, or no detail at all on if or how the RT programme was progressed across the intervention. While these studies included detail on the initial RT prescription characteristics, no information was available for how the training was progressed thereafter. For example, Ohira $e t a l^{27}$ did not specifically describe any progression of the RT stimulus across a 24-week intervention, whereas Schmidt et al ${ }^{64}$ included the description of 'any increase in intensity was based on the Borg scale' as their specific reference to progression that was implemented during the RT intervention. The principle of progression is critical in the design of an exercise programme to ensure continued development. The body can quickly adapt to a given exercise stress; thus, to see continued improvements in a desired outcome, the training stimulus must be gradually increased. Previous reviews have found the principle of progression to be reported more often in RT interventions than in aerobic exercise interventions. ${ }^{19}{ }^{20}$ It is possible that inadequate implementation of the progression principle could minimise the effect of the RT intervention leading to misinterpretation of null findings as evidence of a lack of efficacy of the intervention rather than a non-optimal design of the load and volume comprising the RT stimulus. Consequently, the reporting of the principle of progression is critical for appropriate evaluation and replication of the RT intervention.

An exercise stimulus serves to create biological stress, disrupting cellular and systemic environments. ${ }^{85-88}$ This disruption results in a multisystem biological stress response, whereby the body adapts to the stress in an attempt to withstand future perturbations. ${ }^{85-88}$ Indeed, strategically dosed, chronic RT can result in an array of physiological adaptations and improvements in relevant clinical and patient-reported outcomes. However, the exercise stimuli must be sufficient to stress the system, yet balanced with the recognition that excessive stress can disrupt homoeostasis to a point where maladaptation occurs, increasing 


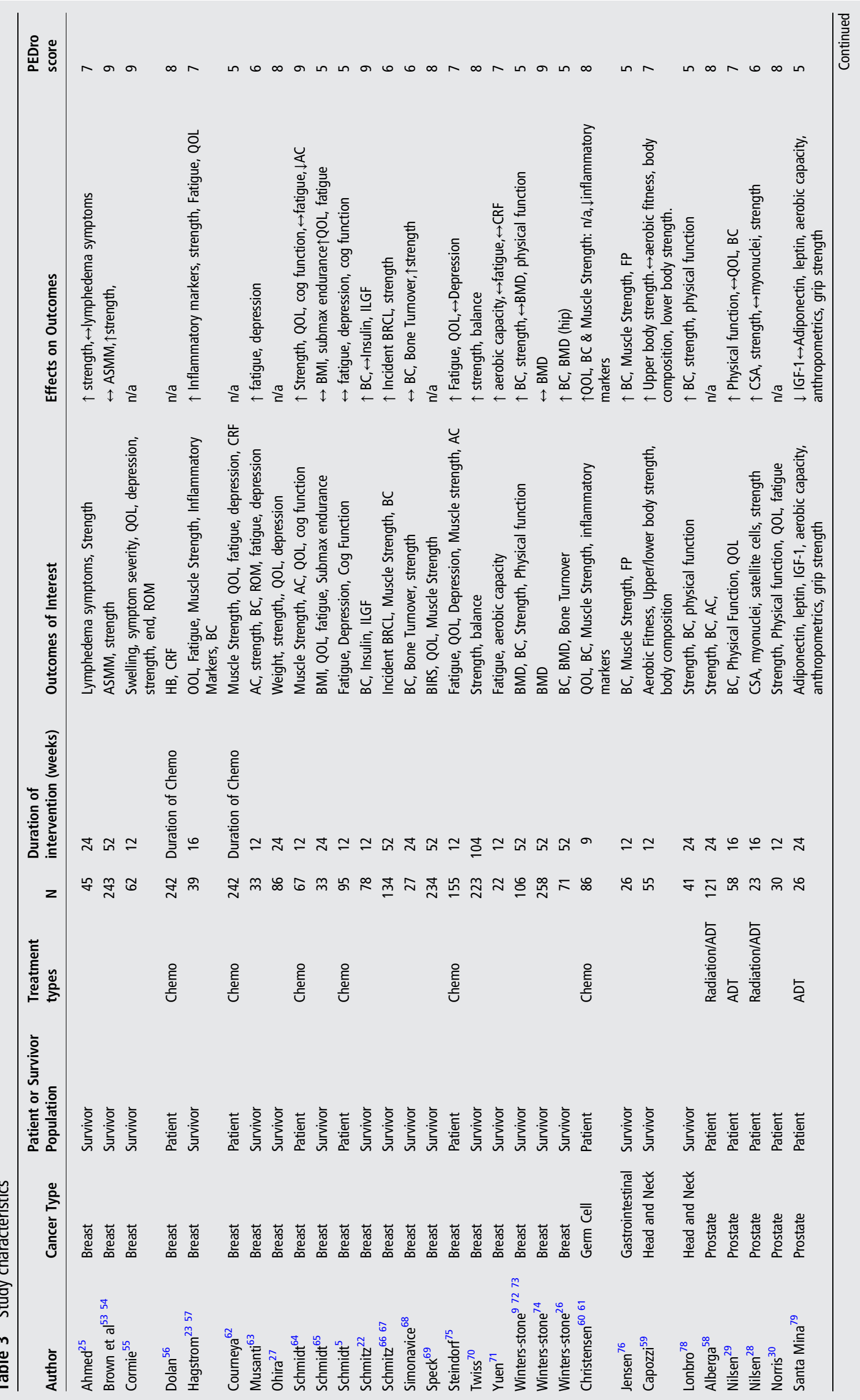




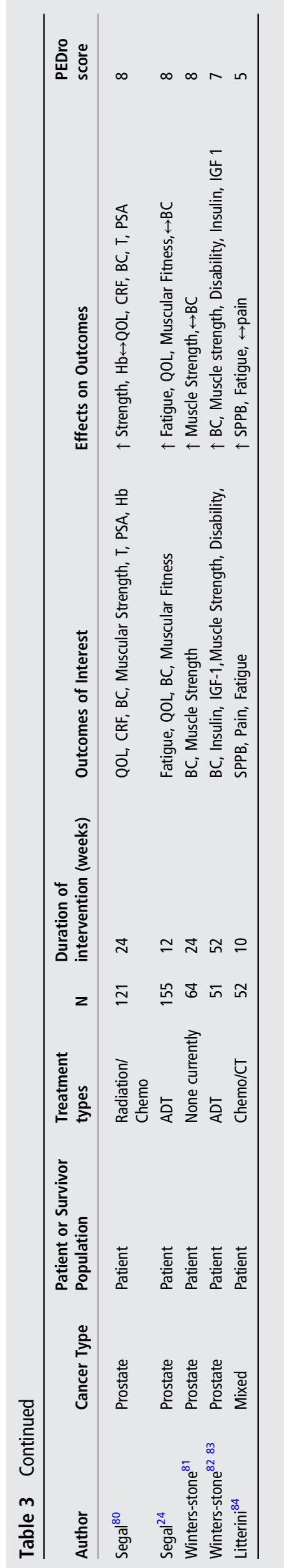

the fatigue and the risk of injury. ${ }^{87} 8990$ The detailed reporting of progression and overload used in the exercise prescription process is an integral consideration in the design and delivery of RT interventions.

Vague descriptions of the exercise stimulus and application of progression can lead to misinterpretation and delivery in clinical or community settings. This can result in an inefficient exercise prescription, along with the potential for injury due to overtraining or misapplication of a training stimulus. Moreover, the incorporation and reporting of these training principles are critical to avoid inaccurate conclusions about a negative trial outcome that may be more attributable to the intervention design than a lack of efficacy.

RT prescription is a complex art, involving the manipulation of numerous variables. Thus, explicit description of the exercise intervention is imperative to the evaluation and replication of studies. $^{91-93}$ In a recent systematic review of 73 systematic reviews, Slade and Keating ${ }^{94}$ reported that the majority of reviews were unable to appropriately describe the exercise interventions included because the required information was poorly described or not described at all. In an attempt to rectify this, a standardised method for reporting exercise programmes was developed by Slade et al, ${ }^{91}$ comprehensively detailing critical components of an intervention such as the location, level of supervision, detailed description of exercises, dose and progression, individualisation and instructor qualification/experience. Certainly, the inclusion of an exercise reporting grid would dramatically improve the ability to accurately evaluate, replicate and implement interventions. We recommend a standardised exercise-reporting tool, such as the one outlined by Slade et $a l,{ }^{91}$ to be considered by researchers and journals to enhance the quality of exercise reporting in oncology trials.

\section{RT prescription characteristics}

In addition to evaluating the implementation of RT principles, determining the proportion of RT interventions that explicitly reported the relevant RT prescription components was also a primary objective of this systematic review. The present findings revealed that the majority of studies explicitly reported the relevant RT prescription components. Although the initial RT stimulus components were consistently reported in appropriate detail in virtually all the trials, details of if or how the initial RT stimulus components were modified across the trial were not consistently addressed. Specifically, the extent to which RT components such as load or volume were modified during the intervention to promote progression or overload were not described in detail. As noted previously, this is a primary contributing factor to the inability to determine if the principles of progression and overload were incorporated and not reported, or whether they were not incorporated at all.

It should be noted that the most common exercise prescription $(\sim 50 \%)$ implemented in the studies included in this review were based on of the American College of Sports Medicine (ACSM) recommendations for RT. The ACSM guidelines recommend 2-3 days/week of RE, focusing on large muscle groups, $60-70 \% 1 \mathrm{RM}, 1-3$ sets of $8-12$ repetitions. ${ }^{12-15}$ Of the 33 studies included in this review, 18 used these guidelines, focusing on stimulating the whole body during each session. Given the benefits accompanying RT in trials implementing the ACSM recommendations, these guidelines appear to be effective at improving clinically relevant patient-reported outcomes including muscular strength, quality of life, fatigue, etc.

Nonetheless, it is also important to recognise there is considerable variability in the response and adaptation to a specific 
training stimulus in an apparently healthy population. ${ }^{95}$ Thus, it is possible the heterogeneity in the response to a given training stimulus may be compounded in an individual with cancer as a function of multiple aspects including, but not limited to, their present status in the cancer control continuum (pretreatment, during treatment or post-treatment), cancer site, treatment (type, dose, duration), age, comorbidities, activity level and body composition. For example, the exercise prescription for a patient with breast cancer undergoing chemotherapy will require modification to account for range of motion limitations of the upper body as a result of a port inserted for chemotherapy infusion, or surgery to remove a tumour. This may differ from a patient with lung cancer having radiation to the chest wall and is recovering from partial lung lobectomy. Indeed, a prostate cancer survivor on chronic androgen deprivation therapy will require a unique prescription to account for castrate levels of anabolic hormones. Additionally, any single RT prescription is unlikely to represent the optimal stimulus for all patients or yield a uniform magnitude of improvement across all outcomes relevant for treatment of patients with cancer. Accordingly, while there will be variability in the implementation and responses to RT in different patient populations with cancer across the cancer control continuum, there will also be heterogeneity in the responses to RT within patients of the same cancer type and/or diagnosis. Accordingly, RT prescriptions that personalise the intervention stimulus to the individual needs, preferences and tolerances are critical to the efficacy of integrating RT in the supportive care efforts. This emphasises the highly individualised nature of exercise prescription in an oncology setting, and the need to investigate different doses, frequencies, duration or load of RT.

The current national guidelines on physical activity in a patient population with cancer highlight the progress that the field of exercise oncology has made since the first published research 30 years ago. However, despite the advances in research and increased international recognition of the importance of integrating exercise in oncology treatment, the findings of the present review demonstrate the inconsistency with which fundamental principles of RT have been implemented in randomised controlled RT trials during and following cancer treatment. Accordingly, the findings suggest current approaches to RT prescription can be characterised as basic and potentially even underdeveloped. ${ }^{16}$

Conversely, several studies included in this review warrant attention for their novel design and exercise prescription approach. Hagstrom et al (2015) employed the traditional ACSM guidelines in breast cancer survivors (with 2 days a week of machine-based exercises: leg extension, machine bench press, back extension) for the first 8 weeks of the study. Participants then transitioned to free weight, barbell compound exercises (squat, deadlift, bench press, etc) for the second 8 weeks. ${ }^{23}$ It is understood that free weight exercises stimulate muscle tissue to a greater degree than machine-based exercises. ${ }^{96} 97$ Thus, Hagstrom's transition to free weight exercises represents an attempt to progress the exercise prescription and stimulate muscle tissue to a greater degree. Indeed, participants who participated in the RT intervention increased leg press strength by $40 \mathrm{~kg}$ in 16 weeks. When compared with a $20 \mathrm{~kg}$ increase in leg press strength in another study that used the ACSM guidelines for a year, ${ }^{66}$ these findings highlight the critical importance of integrating progressive overload in the design and delivery of RT interventions and also underscore how the strategic manipulation of training variables can optimise the effects of RT in producing meaningful improvements in clinically relevant outcomes in patients with cancer and cancer survivors.
Nilsen et al used an undulating (more frequent variations in intensity) model of RT in patients with prostate cancer, alternating between a $10 \mathrm{RM}, 6 \mathrm{RM}$ and $80-90 \% 10 \mathrm{RM}$ on day 1,2 and 3 of their programme, respectively. Participants in the RT group exhibited improvements in muscle strength and crosssectional area (CSA) following 16 weeks of the intervention. Unfortunately, the lack of a comparison intervention group using a more traditional approach to RT prescription precludes the opportunity to evaluate the comparative efficacy of the two RT approaches. There is substantial evidence supporting higher intensity exercise at eliciting greater muscle morphological and neuromuscular changes, along with strength, which ultimately facilitate greater improvements in functional mobility and quality of life. ${ }^{98} 99$ Thus, the usage of an undulating RT model, with higher intensity exercise is a novel, progressive one in the exercise oncology field that warrants further investigation.

Norris et al examined the effects of RT frequency on strength, physical function and psychosocial outcomes in patients with prostate cancer. The researchers compared 12 weeks of different RT training frequency (2 days/week vs 3 days/week). The addition of the extra training session resulted in $\sim 50 \%$ extra training volume. Potentially meaningful effects were found for lower body strength and select physical function outcomes in the 3-day/ week group. ${ }^{30}$ While the addition of an extra day of RT, with $\sim 50 \%$ additional training volume shows initial promise, It is unclear if greater differences between training frequency and volume would emerge after a longer intervention period.

These recent studies signify a novel area of the field of exercise oncology, where there is more interest in the design of the RT interventions, with the aim to determine the extent to which systematic, strategic manipulation of dose, sequencing and progression of RT can optimise improvements in clinically relevant outcomes during and following cancer treatment.

\section{Practical application}

Several position stands and roundtables have provided initial guidelines for implementing RT in a patient population with cancer with site-specific precautions. ${ }^{12-15}$ Indeed, detailed guidelines of RT prescription among different cancer types, at different treatment time points, is beyond the scope of this review. However, we have outlined a few brief guidelines below:

- A comprehensive fitness evaluation is strongly recommended prior to beginning a RT programme to identify potential phys$\mathrm{ical} /$ psychosocial limitations to exercise. For example, a patient with prostate cancer may have difficulty in the seated position in the weeks following surgery, whereas a patient with breast cancer may need alterations in upper body exercise prescription to account for range of motion difficulty following surgery.

- A minimum of 2 days/week of RT is recommended for most patient populations, with the aim of maintaining/improving lean body mass throughout and following treatment. A total body approach of 6-8 exercises per session aimed at stimulating large muscle groups (leg press, leg curl, leg extension, Romanian deadlift, chest press, shoulder press, lat pulldown, seated row) is advocated.

- The incorporation and reporting of key RT principles is absolutely critical to the continued improvement in clinically relevant outcomes. Progression and overload should be incorporated on an individual basis according to patient response.

- Future studies should seek to investigate exercise prescription characteristics outside those proposed by ACSM, or compare these to traditional guidelines in an attempt to determine if an optimal dose-response relationship exists between exercise and target outcomes. 
- A standardised exercise-reporting tool can be incorporated into future interventions to improve the ability to appropriately evaluate and replicate interventions.

Future studies grounded in scientific RT principles, examining different RT interventions, for longer duration, with transparency in reporting of the stimulus components of the RT intervention will ensure continued progress in the field. This, combined with current knowledge, can serve to advance the field and ensure correct interpretation and application of the intervention in a clinical and community setting.

\section{Limitations}

Although we believe the present findings have meaningful implications for future RT interventions among patient populations with cancer, there are several limitations that should be acknowledged. First, the primary purpose of this review was to evaluate the extent to which RT principles were implemented in RT trials, not to explore if implementation of these principles influenced the effect of the RT interventions on relevant clinical or patientreported outcomes. We contend that addressing the proportion of trials that implement RT principles and explicitly report the RT stimulus components is a critical initial step in advancing the knowledge of and application of RT across the cancer control continuum. Nonetheless, we recognise the importance of determining if implementation of RT principles impacts the benefits for patient populations with cancer. As more RCTs implementing the RT principles among patients with cancer and cancer survivors emerge, future reviews using standardised meta-analytic procedures to examine the influence of implementing these training principles on relevant outcomes are warranted.

The majority of studies included in this review were conducted in breast and prostate cancer. Thus, the paucity of studies conducted in patient populations with other types of cancer limit the generalisability of our review across the cancer control continuum. As studies investigating exercise in less common forms of cancer slowly emerge, the recommendations for RT can be expanded to a broader range of populations.

\section{SUMMARY/CONCLUSIONS}

Results of the present review suggest there is meaningful variability in the implementation of the key RT principles of progression and overload across RT trials among patients with cancer and cancer survivors. Additionally, whereas the majority of studies explicitly reported the relevant initial RT prescription components, the extent to which RT components such as load or volume were modified during the intervention to promote progression or overload were not described in detail. Additionally, the majority of studies used RT prescription characteristics that fell within ACSM's guidelines. ${ }^{15}$

\section{What are the findings?}

- The majority of studies apply a largely generic resistance training (RT) prescription to a considerably heterogeneous population.

- More recently, studies have begun to investigate different doses, frequencies, timing and progression of RT in the patient population with cancer.

- There is a critical need for RT interventions to rigorously apply the principles of RT, along with clear reporting of intervention characteristics.

\section{How might it impact on clinical practice in the future?}

- Practitioners are encouraged to use central tenets of RT prescription when designing and implementing programmes in a patient population with cancer, with special attention being paid to principles of progression and overload.

- These findings may encourage practitioners to experiment with different doses, frequencies and progression of RT to provide an optimal training stimulus in the patient population with cancer.

- While the safety and efficacy of RT has been demonstrated during and after cancer in a variety of patient populations with cancer, there has been considerably less research conducted in less common cancers (ie, germ cell, head and neck). Accordingly, practitioners should err on the side of caution with RT prescription and may need to progress at a slower rate according to patient response.

Correction notice This article has been corrected since it was published Online First. Table 3 has been replaced with a new version.

Contributors CMF and BCF conceptualised the review. CMF and PNH performed the review. CMF, BCF and PNH wrote the manuscript.

Competing interests None declared.

Provenance and peer review Not commissioned; externally peer reviewed.

\section{REFERENCES}

1 Collado-Hidalgo A, Bower JE, Ganz PA, et al. Inflammatory biomarkers for persistent fatigue in breast cancer survivors. Clin Cancer Res 2006;12:2759-66.

2 Berger AM, Mooney K, Alvarez-Perez A, et al. Cancer-related fatigue, version 2.2015. J Natl Compr Canc Netw 2015;13:1012-39.

3 Peeters KCMJ, van de Velde $\mathrm{CJH}$, Leer JWH, et al. Late side effects of short-course preoperative radiotherapy combined with total mesorectal excision for rectal cancer: increased bowel dysfunction in irradiated patients--a Dutch colorectal cancer group study. J Clin Oncol 2005;23:6199-206.

4 Shapiro $\mathrm{CL}$, Recht A. Side effects of adjuvant treatment of breast cancer. $N$ Engl J Med 2001;344:1997-2008.

5 Schmidt ME, Wiskemann J, Armbrust $P$, et al. Effects of resistance exercise on fatigue and quality of life in breast cancer patients undergoing adjuvant chemotherapy: a randomized controlled trial. Int J Cancer 2014

6 Schneider CM, Hseih CC, Sprod LK, et al. Cancer treatment-induced alterations in muscular fitness and quality of life: the role of exercise training. Ann Oncol 2007; 18:1957-62.

7 Jones SB, Thomas GA, Hesselsweet SD, et al. Effect of exercise on markers of inflammation in breast cancer survivors: the Yale exercise and survivorship study. Cancer Prev Res (Phila) 2013;6:109-18.

8 Courneya KS, McKenzie DC, Mackey JR, et al. Effects of exercise dose and type during breast cancer chemotherapy: multicenter randomized trial. J Nal Cancer Inst 2013;105:1821-32.

9 Winters-Stone KM, Dobek J, Bennett JA, et al. The effect of resistance training on muscle strength and physical function in older, postmenopausal breast cancer survivors: a randomized controlled trial. J Cancer Surviv 2012;6:189-99.

10 Irwin ML, Varma K, Alvarez-Reeves M, et al. Randomized controlled trial of aerobic exercise on insulin and insulin-like growth factors in breast cancer survivors: the Yale Exercise and Survivorship study. Cancer Epidemiol Biomarkers Prev 2009;18:306-13.

11 Fairman CM, Kendall KL, Harris BS, et al. Utilization of an anti-gravity treadmill in a physical activity program with female breast cancer survivors: a pilot study. Int J Exerc Sci 2016;9:101-9.

12 Schmitz KH, Courneya KS, Matthews C. American College of Sports Medicine roundtable on exercise guidelines for cancer survivors. Med Sci Sports Exerc 2010;42:1409-26.

13 Campbell A, Stevinson C, Crank H. The BASES Expert Statement on exercise and cancer survivorship. J Sports Sci 2012;30:949-52.

14 Hayes SC, Spence RR, Galvão DA, et al. Australian Association for Exercise and Sport Science position stand: optimising cancer outcomes through exercise. 2009;12:428-34.

15 Schmitz KH. Exercise prescription and programming adaptations. In: Wilkins LW, ed. ACSM's guide to exercise and cancer survivorship. Philadelphia, PA: 2012.

16 Sasso JP, Eves ND, Christensen JF, et al. A framework for prescription in exercise-oncology research. J Cachexia Sarcopenia Muscle 2015;6:115-24. 
17 Fleck SJ, Kraemer WJ, eds. Developing the individualized resistance training workout. Designing resistance training programs. 4th edn. Champaign, IL, 2014.

18 Wilkins LW, ed. General principles of exercise prescription. ACSM's guide to exercise and cancer survivorship. Philadelphia, PA, 2014.

19 Winters-Stone KM, Neil SE, Campbell KL. Attention to principles of exercise training: a review of exercise studies for survivors of cancers other than breast. $\mathrm{Br}$ J Sports Med 2014;48:987-95.

20 Campbell KL, Neil SE, Winters-Stone KM. Review of exercise studies in breast cancer survivors: attention to principles of exercise training. $\mathrm{Br} J$ Sports Med 2012:46:909-16.

21 Maher CG, Sherrington C, Herbert RD, et al. Reliability of the PEDro scale for rating quality of randomized controlled trials. Phys Ther 2003;83:713-21.

22 Schmitz KH, Ahmed RL, Hannan PJ, et al. Safety and efficacy of weight training in recent breast cancer survivors to alter body composition, insulin, and insulin-like growth factor axis proteins. Cancer Epidemiol Biomarkers Prev 2005;14:1672-80.

23 Hagstrom AD, Marshall PWM, Lonsdale $C$, et al. Resistance training improves fatigue and quality of life in previously sedentary breast cancer survivors: a randomised controlled trial. Eur J Cancer Care (Engl) 2015.

24 Segal RJ, Reid RD, Courneya KS, et al. Resistance exercise in men receiving androgen deprivation therapy for prostate cancer. J Clin Oncol 2003;21:1653-9.

25 Ahmed RL, Thomas W, Yee $\mathrm{D}$, et al. Randomized controlled trial of weight training and lymphedema in breast cancer survivors. J Clin Oncol 2006;24:2765-72.

26 Winters-Stone KM, Dobek J, Nail LM, et al. Impact + resistance training improves bone health and body composition in prematurely menopausal breast cancer survivors: a randomized controlled trial. Osteoporos Int 2013;24:1637-46.

27 Ohira T, Schmitz KH, Ahmed RL, et al. Effects of weight training on quality of life in recent breast cancer survivors: the Weight Training for Breast Cancer Survivors (WTBS) study. Cancer 2006;106:2076-83.

28 Nilsen TS, Thorsen L, Fosså SD, et al. Effects of strength training on muscle cellular outcomes in prostate cancer patients on androgen deprivation therapy. Scand J Med Sci Sports 2015.

29 Nilsen TS, Raastad T, Skovlund E, et al. Effects of strength training on body composition, physical functioning, and quality of life in prostate cancer patients during androgen deprivation therapy. Acta Oncol 2015;54:1805-13.

30 Norris MK, Bell GJ, North $\mathrm{S}$, et al. Effects of resistance training frequency on physical functioning and quality of life in prostate cancer survivors: a pilot randomized controlled trial. Prostate Cancer Prostatic Dis 2015:18:281-7.

31 Hanson ED, Sheaff AK, Sood S, et al. Strength training induces muscle hypertrophy and functional gains in black prostate cancer patients despite androgen deprivation therapy. J Gerontol A Biol Sci Med Sci 2013;68:490-8.

32 Katz E, Dugan NL, Cohn JC, et al. Weight lifting in patients with lower-extremity lymphedema secondary to cancer: a pilot and feasibility study. Arch Phys Med Rehabil 2010;91:1070-6.

33 Peddle-McIntyre CJ, Bell G, Fenton D, et al. Feasibility and preliminary efficacy of progressive resistance exercise training in lung cancer survivors. Lung Cancer 2012;75:126-32

34 Peddle-McIntyre CJ, Bell G, Fenton D, et al. Changes in motivational outcomes after a supervised resistance exercise training intervention in lung cancer survivors. Cancer Nurs 2013;36:E27-35.

35 Winters-Stone KM, Li F, Horak F, et al. Comparison of tai chi vs. strength training for fall prevention among female cancer survivors: study protocol for the GET FIT trial. BMC Cancer 2012;12:577.

36 Cormie P, Galvão DA, Spry N, et al. Functional benefits are sustained after a program of supervised resistance exercise in cancer patients with bone metastases: longitudinal results of a pilot study. Support Care Cancer 2014;22:1537-48.

37 Battaglini C, Bottaro M, Dennehy C, et al. The effects of an individualized exercise intervention on body composition in breast cancer patients undergoing treatment. Sao Paulo Med J 2007;125:22-8.

38 Galvão DA, Taaffe DR, Spry N, et al. Acute versus chronic exposure to androgen suppression for prostate cancer: impact on the exercise response. J Urol 2011;186:1291-7.

39 Hwang $\mathrm{CL}$, Yu CJ, Shih JY, et al. Effects of exercise training on exercise capacity in patients with non-small cell lung cancer receiving targeted therapy. Support Care Cancer 2012;20:3169-77.

40 Sellar CM, Bell GJ, Haennel RG, et al. Feasibility and efficacy of a 12-week supervised exercise intervention for colorectal cancer survivors. Appl Physiol Nutr Metab 2014;39:715-23.

41 Quist M, Rørth M, Zacho M, et al. High-intensity resistance and cardiovascular training improve physical capacity in cancer patients undergoing chemotherapy. Scand J Med Sci Sports 2006;16:349-57.

42 Quist M, Adamsen L, Rørth $\mathrm{M}$, et al. The impact of a multidimensional exercise intervention on physical and functional capacity, anxiety, and depression in patients with advanced-stage lung cancer undergoing chemotherapy. Integr Cancer Ther 2015:14:341-9.

43 Arbane G, Douiri A, Hart N, et al. Effect of postoperative physical training on activity after curative surgery for non-small cell lung cancer: a multicentre randomised controlled trial. Physiotherapy 2014;100:100-7.
44 Hojan K, Kwiatkowska-Borowczyk E, Leporowska E, et al. Physical exercise for functional capacity, blood immune function, fatigue and quality of life in high-risk prostate cancer patients during radiotherapy. A prospective, randomised clinical study. Eur J Phys Rehabil Med 2016.

45 De Backer IC, Van Breda E, Vreugdenhil A, et al. High-intensity strength training improves quality of life in cancer survivors. Acta Oncol 2007;46:1143-51.

46 Schmidt ME, Meynköhn A, Habermann N, et al. Resistance exercise and inflammation in breast cancer patients undergoing adjuvant radiation therapy: mediation analysis from a randomized, controlled intervention trial. Int J Radiat Oncol Biol Phys 2016;94:329-37.

47 Hansen PA, Dechet CB, Porucznik CA, et al. Comparing eccentric resistance exercise in prostate cancer survivors on and off hormone therapy: a pilot study. PM $R$ 2009:1:1019-24

48 LaStayo PC, Marcus RL, Dibble LE, et al. Eccentric exercise versus usual-care with older cancer survivors: the impact on muscle and mobility--an exploratory pilot study. BMC Geriatr 2011;11:5.

49 De Backer IC, Vreugdenhil G, Nijziel MR, et al. Long-term follow-up after cancer rehabilitation using high-intensity resistance training: persistent improvement of physical performance and quality of life. Br J Cancer 2008;99:30-6.

50 Courneya KS, Segal RJ, Gelmon K, et al. Six-month follow-up of patient-rated outcomes in a randomized controlled trial of exercise training during breast cancer chemotherapy. Cancer Epidemiol Biomarkers Prev 2007;16:2572-8.

51 McNeely ML, Parliament MB, Seikaly $H$, et al. Sustainability of outcomes after a randomized crossover trial of resistance exercise for shoulder dysfunction in survivors of head and neck cancer. Physiother Can 2015;67:85-93.

52 LaStayo PC, Larsen S, Smith S, et al. The feasibility and efficacy of eccentric exercise with older cancer survivors: a preliminary study. J Geriatr Phys Ther 2010;33:135-40.

53 Brown JC, Schmitz KH. Weight lifting and appendicular skeletal muscle mass among breast cancer survivors: a randomized controlled trial. Breast Cancer Res Treat 2015;151:385-92.

54 Brown JC, Troxel AB, Schmitz KH. Safety of weightlifting among women with or at risk for breast cancer-related lymphedema: musculoskeletal injuries and health care use in a weightlifting rehabilitation trial. Oncologist 2012;17:1120-8.

55 Cormie P, Pumpa K, Galvão DA, et al. Is it safe and efficacious for women with lymphedema secondary to breast cancer to lift heavy weights during exercise: a randomised controlled trial. J Cancer Surviv 2013;7:413-24.

56 Dolan LB, Gelmon K, Courneya KS, et al. Hemoglobin and aerobic fitness changes with supervised exercise training in breast cancer patients receiving chemotherapy. Cancer Epidemiol Biomarkers Prev 2010;19:2826-32.

57 Hagstrom AD, Marshall PWM, Lonsdale $C$, et al. The effect of resistance training on markers of immune function and inflammation in previously sedentary women recovering from breast cancer: a randomized controlled trial. Breast Cancer Res Treat 2016;155:471-82.

58 Alberga AS, Segal RJ, Reid RD, et al. Age and androgen-deprivation therapy on exercise outcomes in men with prostate cancer. Support Care Cancer 2012;20:971-81

59 Capozzi LC, McNeely ML, Lau HY, et al. Patient-reported outcomes, body composition, and nutrition status in patients with head and neck cancer: results from an exploratory randomized controlled exercise trial. Cancer 2016.

60 Christensen JF, Tolver A, Andersen JL, et al. Resistance training does not protect against increases in plasma cytokine levels among germ cell cancer patients during and after chemotherapy. J Clin Endocrinol Metab 2014;99:2967-76.

61 Christensen JF, Jones LW, Tolver A, et al. Safety and efficacy of resistance training in germ cell cancer patients undergoing chemotherapy: a randomized controlled trial. Br J Cancer 2014;111:8-16.

62 Courneya KS, Segal RJ, Mackey JR, et al. Effects of aerobic and resistance exercise in breast cancer patients receiving adjuvant chemotherapy: a multicenter randomized controlled trial. J Clin Oncol 2007;25:4396-404.

63 Musanti R. A study of exercise modality and physical self-esteem in breast cancer survivors. Med Sci Sports Exerc 2012;44:352-61.

64 Schmidt T, Weisser B, Dürkop J, et al. Comparing endurance and resistance training with standard care during chemotherapy for patients with primary breast cancer. Anticancer Res 2015;35:5623-9.

65 Schmidt T, Weisser B, Jonat W, et al. Gentle strength training in rehabilitation of breast cancer patients compared to conventional therapy. Anticancer Res 2012:32:3229-33.

66 Schmitz $\mathrm{KH}$, Ahmed RL, Troxel $\mathrm{AB}$, et al. Weight lifting for women at risk for breast cancer-related lymphedema: a randomized trial. JAMA 2010;304: 2699-705.

67 Schmitz KH, Ahmed RL, Troxel A, et al. Weight lifting in women with breast-cancer-related lymphedema. N Engl J Med 2009;361:664-73.

68 Simonavice E, Liu PY, Ilich JZ, et al. The effects of a 6-month resistance training and dried plum consumption intervention on strength, body composition, blood markers of bone turnover, and inflammation in breast cancer survivors. Appl Physiol Nutr Metab 2014:39:730-9.

69 Speck RM, Gross CR, Hormes JM, et al. Changes in the Body Image and Relationship Scale following a one-year strength training trial for breast cancer survivors with or at risk for lymphedema. Breast Cancer Res Treat 2010;121:421-30. 
70 Twiss JJ, Waltman NL, Berg K, et al. An exercise intervention for breast cancer survivors with bone loss. J Nurs Scholarsh 2009;41:20-7.

71 Yuen HK, Sword D. Home-based exercise to alleviate fatigue and improve functional capacity among breast cancer survivors. J Allied Health 2007:36:e257-75.

72 Winters-Stone KM, Leo MC, Schwartz A. Exercise effects on hip bone mineral density in older, post-menopausal breast cancer survivors are age dependent. Arch Osteoporos 2012;7:301-6.

73 Winters-Stone KM, Dobek J, Nail L, et al. Strength training stops bone loss and builds muscle in postmenopausal breast cancer survivors: a randomized, controlled trial. Breast Cancer Res Treat 2011;127:447-56.

74 Winters-Stone KM, Laudermilk M, Woo K, et al. Influence of weight training on skeletal health of breast cancer survivors with or at risk for breast cancer-related lymphedema. J Cancer Surviv 2014;8:260-8.

75 Steindorf K, Schmidt ME, Klassen O, et al. Randomized, controlled trial of resistance training in breast cancer patients receiving adjuvant radiotherapy: results on cancer-related fatigue and quality of life. Ann Oncol 2014;25:2237-43.

76 Jensen W, Baumann FT, Stein A, et al. Exercise training in patients with advanced gastrointestinal cancer undergoing palliative chemotherapy: a pilot study. Support Care Cancer 2014;22:1797-806.

77 Lønbro S, Dalgas $U$, Primdahl $H$, et al. Feasibility and efficacy of progressive resistance training and dietary supplements in radiotherapy treated head and neck cancer patients--the DAHANCA 25A study. Acta Oncol 2013;52:310-18.

78 Lønbro S, Dalgas U, Primdahl $\mathrm{H}$, et al. Progressive resistance training rebuilds lean body mass in head and neck cancer patients after radiotherapy--results from the randomized DAHANCA 25B trial. Radiother Oncol 2013;108:314-19.

79 Santa Mina D, Connor MK, Alibhai SMH, et al. Exercise effects on adipokines and the IGF axis in men with prostate cancer treated with androgen deprivation: a randomized study. Can Urol Assoc J 2013:7:E692-8.

80 Segal RJ, Reid RD, Courneya KS, et al. Randomized controlled trial of resistance or aerobic exercise in men receiving radiation therapy for prostate cancer. I Clin Oncol 2009;27:344-51

81 Winters-Stone KM, Lyons KS, Dobek J, et al. Benefits of partnered strength training for prostate cancer survivors and spouses: results from a randomized controlled trial of the Exercising Together project. I Cancer Surviv 2015;10:1-12.

82 Winters-Stone KM, Dieckmann N, Maddalozzo GF, et al. Resistance exercise reduces body fat and insulin during androgen-deprivation therapy for prostate cancer. Oncol Nurs Forum 2015:42:348-56.

83 Winters-Stone KM, Dobek JC, Bennett JA, et al. Resistance training reduces disability in prostate cancer survivors on androgen deprivation therapy: evidence from a randomized controlled trial. Arch Phys Med Rehabil 2015;96:7-14.
84 Litterini AJ, Fieler VK, Cavanaugh JT, et al. Differential effects of cardiovascular and resistance exercise on functional mobility in individuals with advanced cancer: a randomized trial. Arch Phys Med Rehabil 2013;94:2329-35.

85 de Salles BF, Simão R, Fleck SJ, et al. Effects of resistance training on cytokines. Int I Sports Med 2010;31:441-50.

86 Terjung RL, Clarkson P, Eichner ER, et al. American College of Sports Medicine roundtable. The physiological and health effects of oral creatine supplementation. Med Sci Sports Exerc 2000:32:706-17.

87 Kraemer WJ, Ratamess NA. Hormonal responses and adaptations to resistance exercise and training. Sports Med 2005;35:339-61.

88 Gonzalez AM, Hoffman JR, Stout JR, et al. Intramuscular anabolic signaling and endocrine response following resistance exercise: implications for muscle hypertrophy. Sports Med 2016;46:671-85.

89 Fry AC, Kraemer WJ. Resistance exercise overtraining and overreaching Neuroendocrine responses. Sports Med 1997;23:106-29.

90 Carfagno DG, Hendrix JC. Overtraining syndrome in the athlete: current clinical practice. Curr Sports Med Rep 2014;13:45-51.

91 Slade SC, Dionne CE, Underwood M, et al. Consensus on Exercise Reporting Template (CERT): modified Delphi study. Physical Therapy 2016.

92 Slade SC, Dionne CE, Underwood M, et al. Standardised method for reporting exercise programmes: protocol for a modified Delphi study. BMJ Open 2014;4: e006682.

93 Hoffmann TC, Erueti C, Glasziou PP. Poor description of non-pharmacological interventions: analysis of consecutive sample of randomised trials. BMJ 2013;347: f3755.

94 Slade SC, Keating JL. Exercise prescription: a case for standardised reporting. Br J Sports Med 2012;46:1110-13.

95 Timmons JA. Variability in training-induced skeletal muscle adaptation. J Appl Physiol 2011;110:846-53.

96 Schick EE, Coburn JW, Brown LE, et al. A comparison of muscle activation between a Smith machine and free weight bench press. J Strength Cond Res 2010:24:779-84.

97 Schwanbeck S, Chilibeck PD, Binsted G. A comparison of free weight squat to Smith machine squat using electromyography. J Strength Cond Res 2009:23:2588-91.

98 Seynnes 0 , Fiatarone Singh MA, Hue 0 , et al. Physiological and functional responses to low-moderate versus high-intensity progressive resistance training in frail elders. J Gerontol A Biol Sci Med Sci 2004;59:503-9.

99 Fry AC. The role of resistance exercise intensity on muscle fibre adaptations. Sports Med 2004;34:663-79. 